\title{
Atendimento a tentativas de suicídio por serviço de atenção pré-hospitalar
}

\author{
Pre-hospital attendance to suicide attempts
}

Ana Paula Nogueira de Magalhães', Verônica de Medeiros Alves², Isabel Comassetto ${ }^{3}$, Patrícia Costa Lima Ana Cristina Mancussi e Far0 ${ }^{5}$, Antonio Egidio Nardi ${ }^{6}$

\section{RESUMO}

Objetivo: Investigar as características das vítimas de tentativa de suicídio atendidas em serviço pré-hospitalar e os intervalos de tempo consumidos nessa fase de atendimento. Métodos: Estudo transversal utilizando dados da fase pré-hospitalar de atendimento às vítimas de tentativa de suicídio no município de Arapiraca, no ano de 2011. Para análise dos dados, foram realizados teste exato de Fisher, teste t de Student e regressão logística múltipla.

Resultados: Foram atendidas 80 vítimas de tentativa de suicídio pelo serviço de atenção pré-hospitalar. As mulheres, com idade superior a dos homens, foram as que mais tentaram suicídio ( $n=44,55 \%)$, e a intoxicação por medicamentos foi o método mais utilizado ( $n=44$, $55 \%$ ). As tentativas de suicídio ocorreram com maior frequência no outono ( $n=29,36,25 \%)$, no dia de domingo $(n=18,22,5 \%)$, principalmente no período vespertino $(n=33,41,25 \%)$. 0 tempo gasto para o atendimento pré-hospitalar variou entre 34,4 e 40,5 minutos. As variáveis que estiveram associadas às tentativas de suicídio por sexo foram idade $(p=0,03)$ e tempo de transporte $(p=0,01)$. Conclusão: Foram encontradas diferenças entre os sexos das vítimas de tentativa de suicídio atendidas pelo serviço de atenção pré-hospitalar. As mulheres apresentaram maior idade que os homens e o tempo de transporte foi maior em vítimas do sexo masculino, sugerindo maior gravidade nas tentativas de suicídio cometidas por esse grupo.

\section{ABSTRACT}

Objective: To investigate the characteristics of the victims of suicide attempts treated in pre-hospital services and the time intervals consumed in this phase of care. Methods: Cross-sectional study, which used data from pre-hospital care to victims of suicide attempts occurred in the town of Arapiraca, in 2011. For data analysis, Fisher's exact test, Student's t test and multiple logistic regression were performed. Results: A total of 80 victims of suicide attempts were treated by pre-hospital care service. Women over the age of men were the most attempted suicide victims $(n=44,55 \%)$ and drug intoxication was the most used method

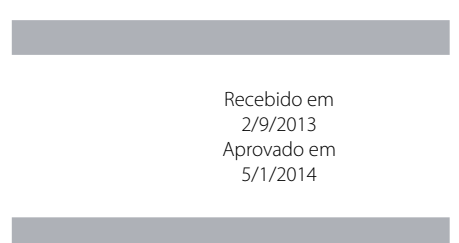

1 Universidade de São Paulo (USP) - campi São Paulo e Ribeirão Preto, Programa Interunidades de Doutoramento em Enfermagem. Universidade Federal de Alagoas (UFAL) - campus Arapiraca.

2 Universidade Federal do Rio de Janeiro (UFRJ), Instituto de Psiquiatria, Programa de Pós-graduação em Saúde Mental. UFAL - campus Arapiraca.

3 USP - campi São Paulo e Ribeirão Preto, Programa Interunidades de Doutoramento em Enfermagem. UFAL, Escola de Enfermagem e Farmácia.

4 UFAL. Prefeitura do município de Viçosa, Alagoas.

5 USP - campi São Paulo e Ribeirão Preto, Programa Interunidades de Doutoramento em Enfermagem. USP, Escola de Enfermagem, Departamento de Enfermagem Médico-Cirúrgica.

6 UFRJ, Instituto de Psiquiatria, Programa de Pós-graduação em Saúde Mental. UFRJ, Instituto de Psiquiatria, Faculdade de Medicina.

Endereço para correspondência: Ana Paula Nogueira de Magalhães

Praça Rozendo Vieira Lima, 330, Cacimbas

57304-500 - Arapiraca, Al, Brasil

E-mail:anapaulanogueira@usp.br 


\section{Keywords}

Suicide attempted, suicide, emergency medical services, epidemiology.
( $n=44,55 \%)$. Suicide attempts occurred more frequently in autumn ( $n=29,36.25 \%)$, on Sunday $(n=18,22.5 \%)$, mainly in the afternoon $(n=33,41.25 \%)$. The time spent in the pre-hospital care varied between 34.4 and 40.5 minutes. The variables that were associated with suicide attempts by sex were: age $(p=0.03)$ and the transportation time $(p=0.01)$. Conclu-

sion: It was found that there were differences between the sexes of the victims of suicide attempts treated by pre-hospital attendance. Women were older than men and the transportation time was higher in male victims, suggesting greater severity in suicide attempts committed by this particular group.

\section{INTRODUÇÃO}

O comportamento suicida é um fenômeno complexo, universal e representa um grande problema de saúde pública em todo o mundo'. Segundo a Organização Mundial da Saúde (OMS), as taxas de suicídio aumentaram 60\% nas últimas décadas, tornando-se a terceira causa de morte entre indivíduos com idade entre 15 e 44 anos $^{2}$.

No Brasil, cerca de quatro habitantes por 100 mil, em média, suicidam-se, e um número difícil de calcular tenta se autoinfligir ${ }^{3}$. Embora as taxas de suicídio sejam consideradas baixas quando comparadas às de outros países², entre 1998 e 2008 o total de suicídios no Brasil representou um aumento de $33,5 \%$, enquanto o crescimento da população do país foi de $17,8 \%$, no mesmo período 4 .

A tentativa de suicídio tem as mesmas características fenomenológicas do suicídio, diferindo deste apenas quanto ao desfecho, que não é fatal ${ }^{5}$. Alguns fatores têm sido associados ao comportamento suicida, como presença de um transtorno mental, antecedentes familiares, uso de álcool, sexo, idade, ausência de um companheiro, estar desempregado ou aposentado, isolamento social e história de abuso sexual na infância ${ }^{5}$. O risco de suicídio aumenta de acordo com o número de tentativas e também está associado a intervalos de tempo menores entre essas tentativas. Entre os pacientes atendidos em setores de emergência por tentativa de autoextermínio, estima-se que de 30\% a 60\% tiveram tentativas prévias e que de $10 \%$ a $25 \%$ tentarão novamente no prazo de um ano?.

Dentre os cuidados iniciais oferecidos às vítimas de tentativa de suicídio, destaca-se o atendimento realizado pelo serviço de atenção pré-hospitalar. Este é definido como o atendimento que procura chegar precocemente à vítima, após ter ocorrido um agravo à sua saúde que possa levar a sofrimento ou mesmo à morte, garantindo atendimento e/ ou transporte adequado a um serviço de saúde integrado ao Sistema Único de Saúde (SUS).

$\mathrm{O}$ atendimento adequado e o tempo decorrido entre a tentativa de suicídio e a admissão hospitalar são fatores extremamente relevantes para reduzir a mortalidade das vítimas? A primeira hora (golden hour) após a ocorrência de uma lesão traumática é considerada o tempo crítico para a instituição do tratamento que modificará o prognóstico, uma vez que até $40 \%$ dos óbitos ocorrem antes da assistência hospitalar ${ }^{8}$.
No Brasil não existem registros sistemáticos das tentativas de suicídio ${ }^{5}$. A utilização de dados advindos dos serviços de atendimento pré-hospitalar pode contribuir para o conhecimento das características sociodemográficas e clínicas dos indivíduos que tentam suicídio, fornecendo informações para o planejamento das ações em saúde e o desenvolvimento de medidas de prevenção mais eficazes.

O presente estudo tem por objetivo investigar as características das vítimas de tentativa de suicídio atendidas em serviço pré-hospitalar e os intervalos de tempo consumidos nessa fase de atendimento.

\section{MÉTODOS}

Trata-se de um estudo transversal que utilizou dados da fase pré-hospitalar de atendimento a vítimas de tentativa de suicídio, no ano de 2011, no município de Arapiraca, localizado na região central de Alagoas, distante $136 \mathrm{~km}$ da capital Maceió, com uma população de 214.067 habitantes ${ }^{9}$. Em termos populacionais e econômicos, é a segunda maior cidade do estado, concentrando mais de 400 mil habitantes em seu entorno imediato.

Os dados foram coletados por meio das fichas de atendimento pré-hospitalar do Serviço de Atendimento Móvel de Urgência (Samu). Foi utilizado um formulário validado por teste piloto contendo as seguintes variáveis: sexo, idade, método utilizado, uso de bebida alcoólica, estação do ano, dia da semana, horário de ocorrência, local do evento, encaminhamento da vítima e óbito.

Os intervalos de tempo considerados no atendimento pré-hospitalar (em minutos) também foram analisados: tempo de resposta (entre o acionamento da equipe e a chegada à cena), tempo de cena (entre a chegada da equipe na cena e a partida para o hospital de destino), tempo de transporte (entre a saída da cena e a chegada ao hospital de destino) e tempo total (entre o acionamento e o hospital).

Para verificar associação, foram utilizados o teste exato de Fisher e o teste t de Student. A análise dos fatores associados às tentativas de suicídio segundo sexo foi realizada por meio de regressão logística múltipla. Foram consideradas candidatas ao modelo multivariado todas as variáveis significativas no nível de 0,20. Em seguida, as variáveis 
menos significativas foram retiradas, uma de cada vez, até permanecerem no modelo somente as variáveis significativas no nível de 0,05. A adequação do modelo foi avaliada pelo teste de Hosmer-Lemeshow. Para a análise dos dados, utilizou-se o pacote estatístico SPSS versão 17.0 (SPSS Inc., (hicago, USA).

Este estudo derivou de uma investigação sobre causas externas em adultos no município de Arapiraca. Por isso, foram excluídos dados referentes a indivíduos menores de 18 anos, não residentes no município em estudo, e fichas de atendimento pré-hospitalar ilegíveis. Em virtude do pequeno número de registros, os métodos de enforcamento e autolesão por objetos cortantes foram agrupados na categoria outros métodos.

Este estudo foi aprovado pelo Comitê de Ética em Pesquisa da Escola de Enfermagem da Universidade de São Paulo (EEUSP), sob parecer no 5.953/2012.

\section{RESULTADOS}

Foram realizados 80 atendimentos por tentativas de suicídio no ano de 2011 pelo serviço de atenção pré-hospitalar, o que corresponde a uma média de um atendimento por tentativa a cada 4,6 dias.

Entre as vítimas atendidas, 44 (55\%) eram do sexo feminino e 36 (45\%), do masculino. A média geral de idade foi de 29,1 anos (desvio-padrão - $d p=9,9$ ), apresentando os homens a média de $26,5(\mathrm{dp}=8,1)$ anos e as mulheres, de $31,3(\mathrm{dp}=10,6)$ anos. Ao comparar a idade entre o sexo das vítimas que tentaram suicídio, observou-se que as mulheres possuíam idade superior a dos homens.

Em relação aos meios utilizados nas tentativas de suicídio, verificou-se que a intoxicação por medicamentos ( $n=$ $44,55 \%)$ e a intoxicação por veneno $(n=18,22,5 \%)$ foram os meios mais comuns (Tabela 1). A intoxicação voluntária por

Tabela 1. Características das tentativas de suicídio atendidas pelo serviço pré-hospitalar segundo sexo (Arapiraca, Alagoas, Brasil, 2011)

\begin{tabular}{|c|c|c|c|c|c|c|c|}
\hline \multirow{3}{*}{ Características } & \multicolumn{4}{|c|}{ Sexo } & \multirow{2}{*}{\multicolumn{2}{|c|}{ Total }} & \multirow{3}{*}{$p$} \\
\hline & \multicolumn{2}{|c|}{ Masculino } & \multicolumn{2}{|c|}{ Feminino } & & & \\
\hline & $\mathrm{N}$ & $\%$ & $\mathrm{~N}$ & $\%$ & N & $\%$ & \\
\hline Método utilizado & & & & & & & 0,053 \\
\hline Intoxicação por medicamentos & 14 & 31,8 & 30 & 68,2 & 44 & 100 & \\
\hline Intoxicação por produtos químicos & 4 & 50,0 & 4 & 50,0 & 8 & 100 & \\
\hline Intoxicação por veneno & 12 & 66,7 & 6 & 33,3 & 18 & 100 & \\
\hline Outros métodos & 5 & 62,5 & 3 & 37,5 & 8 & 100 & \\
\hline Uso de bebida alcoólica & & & & & & & 0,225 \\
\hline Sim & 9 & 60,0 & 6 & 40,0 & 15 & 100 & \\
\hline Não & 5 & 38,5 & 8 & 61,5 & 13 & 100 & \\
\hline Estação do ano & & & & & & & 0,162 \\
\hline Verão & 10 & 55,6 & 8 & 44,4 & 18 & 100 & \\
\hline Outono & 10 & 34,5 & 19 & 65,5 & 29 & 100 & \\
\hline Inverno & 10 & 62,5 & 6 & 37,5 & 16 & 100 & \\
\hline Primavera & 5 & 31,3 & 11 & 68,8 & 16 & 100 & \\
\hline Dia da semana & & & & & & & 0,653 \\
\hline Domingo & 9 & 50,0 & 9 & 50,0 & 18 & 100 & \\
\hline Segunda & 7 & 43,8 & 9 & 53,6 & 16 & 100 & \\
\hline Terça & 2 & 25,0 & 6 & 75,0 & 8 & 100 & \\
\hline Quarta & 2 & 22,2 & 7 & 77,8 & 9 & 100 & \\
\hline Quinta & 6 & 50,0 & 6 & 50,0 & 12 & 100 & \\
\hline Sexta & 3 & 60,0 & 2 & 40,0 & 5 & 100 & \\
\hline Sábado & 6 & 54,5 & 5 & 45,5 & 11 & 100 & \\
\hline Faixa horária & & & & & & & 0,665 \\
\hline $6 \mathrm{~h}$ às $11 \mathrm{~h} 59$ & 8 & 61,5 & 5 & 38,5 & 13 & 100 & \\
\hline $12 \mathrm{~h}$ às $17 \mathrm{~h} 59$ & 14 & 42,4 & 19 & 57,6 & 33 & 100 & \\
\hline $18 \mathrm{~h}$ às $23 \mathrm{~h} 59$ & 11 & 42,3 & 15 & 57,7 & 26 & 100 & \\
\hline $0 h$ às $5 h 59$ & 3 & 37,5 & 5 & 62,5 & 8 & 100 & \\
\hline
\end{tabular}


medicamentos foi duas vezes mais utilizada pelas mulheres que pelos homens. Entretanto, não houve diferença estatística entre sexo e os diferentes métodos.

Entre as vítimas atendidas, identificou-se que 15 (18,7\%) pessoas fizeram uso de álcool. Na maioria dos casos ( $\mathrm{n}=$ $52,65 \%$ ) essa informação foi ignorada. Não foi encontrada significância estatística entre o consumo de álcool por sexo (Tabela 1).

Na tabela 1, observa-se que as tentativas de suicídio ocorreram com maior frequência no outono ( $n=29,36,25 \%)$ e no verão ( $n=18,22,5 \%$ ). $O$ domingo foi o dia da semana com maior número de atendimentos por tentativas de suicídio ( $n=18,22,5 \%)$, seguido pela segunda-feira $(n=16,20,0 \%)$. Nesses dias, o horário predominante para as tentativas de suicídio foi entre 12h e 17h59 ( $n=33,41,25 \%)$.

Em relação aos locais de ocorrência, 54 (67,5\%) tentativas de suicídio ocorreram no domicílio, duas (2,5\%) em uma delegacia e em 24 (30\%) fichas de atendimento pré-hospitalar não constava essa informação.

Quatro (5\%) indivíduos tentaram suicídio mais de uma vez. Os homens em idade jovem (18 a 21 anos) foram os que mais repetiram a tentativa $(n=3,75 \%)$, e o método mais utilizado por eles foi a intoxicação por medicamentos $(n=3$, 75\%). Verificaram-se quatro (5\%) tentativas de suicídio com desfecho letal, sendo dois óbitos em mulheres e dois em homens.

Quanto à média dos tempos consumidos nas fases do atendimento pré-hospitalar (em minutos), observou-se diferença entre sexo e os tempos de transporte e total (Tabela 2). Constatou-se que o tempo de transporte foi significativamente maior nos atendimentos aos homens.

Verificou-se, por meio da análise de regressão logística (Tabela 3), que as variáveis que permaneceram no modelo final com maior associação com o sexo foram tempo de transporte e idade. Quanto maior for o tempo de transporte, maior a chance de a vítima ser do sexo masculino. Em relação à idade, quanto mais velha for a vítima, maior a chance de ela ser do sexo feminino. O teste de adequação de Hosmer-Lemeshow apontou para um bom ajustamento do modelo final $(p=0,250)$.

Tabela 2. Distribuição da média de tempo consumido (em minutos), em cada fase de atendimento às vítimas de tentativa de suicídio, segundo sexo (Arapiraca, Alagoas, Brasil, 2011)

\begin{tabular}{lccc}
\hline \multirow{2}{*}{ Tempos nas fases de atendimento } & \multicolumn{2}{c}{ Sexo } & $p$ \\
\cline { 2 - 3 } & Masculino & Feminino & \\
\hline Tempo de resposta & 15,76 & 15,21 & 0,751 \\
Tempo de cena & 11,35 & 11,03 & 0,823 \\
Tempo de transporte & 10,50 & 7,03 & 0,018 \\
Tempo total & 40,59 & 34,40 & 0,045 \\
\hline
\end{tabular}

Tabela 3. Modelo de regressão logística para vítimas de tentativa de suicídio atendidas em serviço pré-hospitalar, segundo sexo (Arapiraca, Alagoas, Brasil, 2011)

\begin{tabular}{lccc}
\hline Variável & Razão de chance & Intervalo de confiança (95\%) & $p$ \\
\hline Tempo de transporte & 1,22 & $1,04-1,43$ & 0,01 \\
Idade & 0,92 & $0,85-0,99$ & 0,03 \\
\hline
\end{tabular}

A maior parte das vítimas foi encaminhada para unidade de emergência de referência para o atendimento por tentativa de suicídio ( $n=64,80 \%)$; seis $(7,5 \%)$ tiveram alta no próprio local de atendimento; quatro (5\%) recusaram-se a ser encaminhadas para a unidade hospitalar e uma $(1,2 \%)$ foi encaminhada para um hospital psiquiátrico. Uma $(1,2 \%)$ ficha de atendimento apresentou registro indeterminado para essa informação.

\section{DISCUSSÃO}

O presente estudo identificou que uma pessoa foi atendida por tentativa de suicídio a cada 4,6 dias, no serviço pré-hospitalar. Ao levar em consideração que uma em cada três pessoas que tentam o suicídio chega a ser atendida em um serviço médico de urgência ${ }^{10}$, é provável que a quantidade de tentativas seja bem maior.

Muitas tentativas de suicídio podem não chegar ao atendimento hospitalar por serem de baixa complexidade. Isso porque a maioria dos meios suicidas empregados nessa circunstância é pouco violenta, como a ingestão de medicamentos e outras substâncias químicas ${ }^{11}$. Além do grau de gravidade, outros fatores também podem influenciar a procura por um serviço médico após a tentativa de suicídio. Dentre eles, destacam-se: o acesso e a confiança no sistema de saúde, o estigma da população em relação ao comportamento suicida e o medo da criminalização do ato ${ }^{10}$.

As mulheres, adultas jovens, cometeram mais tentativas de suicídio que os homens. Esses achados são consistentes com os da literatura, 10,12,13. Na faixa etária entre 31 e 35 anos, estudos relacionam o comportamento suicida ao grupo de mulheres que não trabalham fora de casa, indicando que a realização de atividades laborais fora do ambiente doméstico promove benefícios à saúde mental desse grupo ${ }^{1,12}$.

Mulheres apresentam comportamento suicida mais frequente e realizam mais tentativas, sendo o método mais utilizado a ingestão de medicamentos e de outras substâncias tóxicas ${ }^{1}$. Esse meio é o eleito por ser menos invasivo e, consequentemente, não afetar a estética ${ }^{14,15}$.

Os homens cometem mais suicídios e preferem métodos que evidenciem sua virilidade, utilizando meios mais letais como enforcamento, arma de fogo e precipitação de lugares elevados ${ }^{1,14-16}$. 
O predomínio de tentativas de suicídio por autointoxicação é reiterado por pesquisas sobre o tema ${ }^{1,17}$. Geralmente, mais de um tipo de substância química, medicamentosa ou não, é utilizado quando o método é a intoxicação voluntária'2.

Em estudo retrospectivo com 206 casos de tentativas de suicídio por intoxicação medicamentosa, os grupos farmacológicos que mais se destacaram foram os tranquilizantes (25,5\%), antidepressivos (17\%) e anticonvulsivos (15\%) ${ }^{12}$. Como a sobredose intencional de medicamentos é considerada o método mais utilizado para a tentativa de suicídio, são necessários uma avaliação cautelosa do estado psíquico e emocional do paciente antes de prescrever tais substâncias ${ }^{12}$ e um acompanhamento contínuo.

Dados obtidos no atual estudo destacam a intoxicação voluntária por veneno, sobretudo por agrotóxicos. A utilização dessas substâncias tem crescido mundialmente e se tornado um grande problema em alguns países asiáticos, latino-americanos e em Portugal ${ }^{15}$.

No município de Arapiraca, os óbitos por ingestão de pesticidas e outros produtos químicos utilizados na agricultura têm chamado a atenção ${ }^{18}$. A disponibilidade dos agrotóxicos pode favorecer atos suicidas não planejados, ocorrendo muitas vezes de maneira impulsiva ${ }^{15}$.

A ingestão de álcool esteve relacionada a 18,7\% das tentativas de suicídio. Entretanto, $65 \%$ das fichas de atendimento pré-hospitalar não apresentaram registros referentes a essa informação. Acredita-se que o uso abusivo do álcool seja maior do que o constatado neste estudo e que a não associação encontrada entre essa variável e a tentativa de suicídio pode estar relacionada à falta de informação. Daí a necessidade de conscientização dos profissionais da saúde quanto ao registro do uso nocivo de álcool para o planejamento de estratégias de cuidado e prevenção.

Em relação à sazonalidade, estudos sugerem que há diferenças entre as variações climáticas e o comportamento suicida ${ }^{19,20}$. Neste estudo, houve maior proporção de tentativas de suicídio no outono, achado semelhante ao de um estudo epidemiológico realizado no interior da Bahia²1.

Nos Estados Unidos, um estudo sobre tentativas de suicídio associadas ao uso de álcool e outras drogas, com vítimas atendidas em serviços de emergência, constatou que o percentual de tentativas no outono foi maior do que no inverno e na primavera ${ }^{19}$. Possivelmente, flutuações no clima e na luz solar causadas pelas estações do ano influenciam o aparecimento de sintomas depressivos ${ }^{19}$.

Quanto aos dias da semana, o domingo, principalmente no período vespertino, foi o dia mais frequente para as tentativas de suicídio. Isso pode ter acontecido pelo fato de as pessoas estarem mais sozinhas, ou ainda pelo fato de as pessoas em tratamento psicológico não terem disponível, de forma adequada, o atendimento especializado nos serviços de saúde. Além disso, no fim de semana, as pessoas estão mais predispostas ao uso de bebida alcoólica, o que pode favorecer a tentativa de suicídio.

Entre os casos de atendimento pré-hospitalar deste estudo, 5\% dos indivíduos tentaram suicídio e repetiram a tentativa em menos de uma semana. Esse achado merece destaque, pois, tanto para aqueles que tentam pela primeira vez quanto para os repetidores, o primeiro ano constitui o período de maior risco'. Essa afirmativa é reforçada pelos resultados obtidos em estudo de coorte retrospectivo com 807 indivíduos, no qual $60 \%$ das mortes por suicídio ocorreram no primeiro ano depois da tentativa-índice!

A maioria das vítimas (80\%) assistidas pelo serviço pré-hospitalar foi encaminhada para o hospital de referência da região. Durante a fase pré-hospitalar, foram realizados os primeiros cuidados para evitar complicações à saúde das vítimas.

A identificação do tempo de duração do atendimento pré-hospitalar é um fator relevante, pois, para pacientes gravemente feridos, os primeiros 60 minutos são cruciais para a manutenção da vida ${ }^{22}$. Neste estudo, o uso do serviço pré-hospitalar permitiu que todas as vítimas de tentativas de suicídio fossem assistidas dentro da referida golden hour.

O tempo consumido no atendimento pré-hospitalar é resultante de um conjunto de fatores que o influenciam, como experiência e capacitação profissional da equipe, condições de tráfego local, dia da semana e período do dia, tipo e número de veículos disponíveis e localização desses veículos ${ }^{23}$.

O presente estudo mostra que o tempo de transporte foi maior em vítimas de tentativa de suicídio do sexo masculino. Possivelmente, esse fato está relacionado à maior gravidade das tentativas de suicídio cometidas pelos homens, pois, de acordo com os dados encontrados na tabela 2, as vítimas do sexo masculino utilizaram métodos considerados mais letais, o que pode ter acarretado a realização de procedimentos mais complexos durante o transporte deles, como a intubação endotraqueal, que necessitam que a viatura seja parada para serem realizados.

O comportamento suicida é um fenômeno complexo e influenciado por vários fatores. Por isso, sua dinâmica precisa ser mais bem compreendida para que sejam propostas estratégias para a prevenção desses agravos e, ao mesmo tempo, adequação das práticas de saúde para melhor assistir suas vítimas. Para isso, é preciso estabelecer um protocolo de intervenções, a ponto de ter impacto sobre a sobrevivência da vítima sem consumir tempo desnecessário ${ }^{24}$.

Os profissionais da atenção pré-hospitalar precisam estar capacitados para lidar com as peculiaridades do comportamento suicida, garantindo abordagem e encaminhamento adequados às vítimas de tentativas de suicídio. É necessário, ainda, que os serviços de saúde estejam articulados, para que haja garantia de acolhimento e continuidade de tratamento, mesmo nos casos menos graves?.

A quantidade de informações faltantes nas fichas de atendimento pré-hospitalar merece destaque, pois o regis- 
tro insuficiente prejudica o conhecimento mais detalhado sobre a distribuição e a magnitude das tentativas de suicídio. Conforme Malvestio ${ }^{25}$, as razões para o mau preenchimento das fichas de atendimento pré-hospitalar podem ser decorrentes da condição de saúde das vítimas encontradas no momento da cena, principalmente quando há casos mais graves, ou podem ser explicadas pela falta de compreensão da importância desse ato pelas equipes de atendimento.

Dessa forma, é fundamental estimular os profissionais de saúde para o preenchimento adequado das fichas de atendimento pré-hospitalar. A obtenção dessas informações pode fornecer subsídios para o planejamento e a avaliação das ações realizadas, permitindo a continuidade da assistência à vítima de tentativa de suicídio no ambiente hospitalar.

As principais limitações deste estudo referem-se à exclusão de indivíduos menores de 18 anos e à utilização de dados secundários incompletos. $\mathrm{O}$ estudo apresentado revela-se importante, por contribuir para a avaliação das características das tentativas de suicídio e suas vítimas, bem como para a identificação da qualidade do serviço de urgência prestado pelas equipes de atenção pré-hospitalar.

\section{CONCLUSÃO}

Os dados apresentados no presente estudo evidenciam diferenças entre as tentativas de suicídio cometidas por homens e mulheres, fato esse que corrobora a literatura e ressalta a importância de se proporem diferentes estratégias de prevenção para cada grupo específico.

Outra questão a ser destacada é o uso frequente de medicamentos e de agrotóxicos para a tentativa de suicídio, o que leva à necessidade de um rigoroso controle sobre a prescrição e a distribuição de medicamentos (principalmente psicofármacos) e sobre a utilização de agrotóxicos (com a utilização de agrotóxicos de menor toxicidade), pois essas são medidas fundamentais para a diminuição das taxas de autoenvenenamento ${ }^{17}$.

Diante disso, é importante destacar que as capacitações nos serviços de atendimento pré-hospitalar devem priorizar, além das habilidades para o atendimento precoce e a manutenção da vida das vítimas, o reconhecimento dos casos de tentativa de suicídio, as principais formas de atuação e o preenchimento adequado das fichas de atendimento pré-hospitalar, pois o conhecimento sobre o comportamento suicida e seus fatores de risco pode contribuir para a qualificação da assistência e para a realização de ações de prevenção, em qualquer nível.

\section{CONTRIBUIÇÕES INDIVIDUAIS}

Ana Paula Nogueira de Magalhães - Contribuiu na concepção e elaboração do estudo, análise e interpretação dos dados, elaboração do artigo e revisão do conteúdo, aprovação da versão final a ser publicada.

Verônica de Medeiros Alves - Contribuiu na análise e interpretação dos dados, elaboração do artigo e revisão do conteúdo, aprovação da versão final a ser publicada.

Isabel Comassetto - Contribuiu na análise e interpretação dos dados, elaboração do artigo e revisão do conteúdo, aprovação da versão final a ser publicada.

Patrícia Costa Lima - Contribuiu na análise e interpretação dos dados, elaboração do artigo e revisão do conteúdo, aprovação da versão final a ser publicada.

Ana Cristina Mancussi e Faro - Contribuiu na concepção do estudo, análise e interpretação dos dados, revisão crítica do conteúdo do artigo, aprovação da versão final a ser publicada.

Antonio Egidio Nardi - Contribuiu na análise e interpretação dos dados, elaboração do artigo e revisão do conteúdo, aprovação da versão final a ser publicada.

\section{CONFLITOS DE INTERESSE}

Os autores declaram não haver conflitos de interesse nem financeiros a serem declarados.

\section{REFERÊNCIAS}

1. Vidal CEL, Gontijo ECDM, Lima LA. Tentativas de suicídio: fatores prognósticos e estimativa do excesso de mortalidade. Cad Saude Publica. 2013;29(1):175-87.

2. World Health Organization. Suicide. Disponivel em: <http://www. who.int/mental_ health/prevention/suicide/suicideprevent/en/>. Acesso em: 24 jun, 2013.

3. Minayo MCS. Conceitos, teorias e tipologias de violência: a violência faz mal à saúde. In: Njaine K, Assis SG, Constantino P, editoras. Impactos da violência na saúde. Rio de Janeiro: Fiocruz; 2010. p. 21-42.

4. Waiselfisz JJ. Mapa da violência 2011: os jovens no Brasil. São Paulo: Instituto Sangari; 2011.

5. Bertolote JM, Mello-Santos C, Botega NJ. Detecção do risco de suicídio nos serviços de emergência psiquiátrica. Rev Bras Psiquiatr. 2010;32:87-95.

6. Brasil. Ministério da Saúde. Portaria no 2.048, de 5 de novembro de 2002. Dispõe sobre 0 regulamento técnico dos sistemas estaduais de urgência e emergência. Diário Oficial da União. Brasília, DF, 6 de novembro de 2002. Seção 1. p. 1.

7. Ladeira RM, Barreto SM. Fatores associados ao uso de serviço de atenção pré-hospitalar por vítimas de acidentes de trânsito. Cad Saude Publica. 2008;24(2):287-94.

8. Itami LT, Faro ACM , Meneghin P, Leite RCBO, Silveira CT. Adultos com fraturas: das implicações funcionais e cirúrgicas à educação em saúde. Rev Esc Enferm USP. 2009;43(2):1238-43.

9. Instituto Brasileiro de Geografia e Estatística (IBGE). Disponível em: <http://www.ibge. gov.br/cidadesat/topwindow.htm?1>. Acesso em: 3 set. 2011

10. Botega NJ, Marín-León L, Oliveira HB, Barros MBA, Silva VF, Dalgalarrondo P. Prevalências de ideação, plano e tentativa de suicídio: um inquérito de base populacional em Campinas, São Paulo, Brasil. Cad Saude Pública. 2009;25(12):2632-8.

11. Vansan GA. Aspectos epidemiológicos comparativos entre tentativas de suicídio e suicídios no município de Ribeirão Preto. J Bras Psiquiatr. 1999:48:209-15.

12. Bernardes SS, Turini CA, Matsuo T. Perfil das tentativas de suicídio por sobredose intencional de medicamentos atendidas por um Centro de Controle de Intoxicaçōes do Paraná, Brasil. Cad Saude Publica. 2010;26:1366-72. 
13. Chen VCH, Tan HKL, Cheng ATA, Chen C-Y, Liao L-R, Stewart $R$, et al. Non-fatal repetition of self-harm: population-based prospective cohort study in Taiwan. Br J Psychiatry. 2010;196:31-5.

14. Abreu KP, Lima MAD, Kohlrausch E, Soares JF. Comportamento suicida: fatores de risco e intervenções preventivas. Rev Eletr Enf. 2010;12(1):195-200.

15. Ajdacic-Gross V, Weiss MG, Ring M, Hepp U, Bopp M, Gutzwiller F, et al. Methods of suicide: international suicide patterns derived from the WHO mortality database. Bull WHO. 2008;86:726-32.

16. Parente ACM, Soares RB, Araújo ARF, Cavalcante IS, Monteiro CFS. Caracterização dos casos de suicídio em uma capital do nordeste brasileiro. Rev Bras Enferm. 2007;60(4):377-81.

17. Macente LB, Santos EG, Zandonade E. Tentativas de suicídio e suicídio em município de cultura pomerana no interior do estado do Espírito Santo. J Bras Psiquiatr. 2009;58(4):238-44.

18. Lima ALA, Bezerra IAD, Sousa LML, Costa MSS, Castro SR, Maciel ACC. Mortalidade por causas externas nos municípios de Arapiraca (AL) e Mossoró (RN) - 1999-2008. Rev Baiana Saúde Pública. 2012;36(1);134-47.

19. Dawn Report: Monthly and seasonal variations in emergency department visits for drugrelated suicide attempts: 2004 to 2008. Disponível em: <http://www.samhsa.gov/ data/2k10/TDR019suicidebyseason/suicidebyseason_HTML.pdf.> Acesso em: 22 maio 2013.
20. Kposowa AJ, D'Auria S. Association of temporal factors and suicides in the United States, 2000-2004. Soc Psychiat Epidemiol. 2010;45:433-45.

21. Souza VS, Alves MS, Silva LA, Lino DCSF, Nery AA, Casotti CA. Tentativas de suicídio e mortalidade por suicídio em um município no interior da Bahia. J Bras Psiquiatr. 2011;60(4):294-300.

22. Sampalis IS, Lavoie A, Williams JI, Mulder DS, Kalina M. Impact of on-site care, prehospital time, and level of in-hospital care on survival in severely injured patients. J Trauma. 1993;34:252-61.

23. Nardoto EML, Diniz JMT, Cunha CEG. Perfil da vítima atendida pelo serviço pré-hospitalar aéreo de Pernambuco. Rev Esc Enferm USP. 2011;45(1):237-42.

24. Malvestio MAA, Souza RMC. Análise do valor predeterminante dos procedimentos da fase pré-hospitalar na sobrevivência das vítimas de trauma. Rev Latino-Am Enfermagem. 2008;16(3):432-8.

25. Malvestio MAA. Predeterminantes de sobrevivência em vítimas de acidentes de trânsito submetidas a atendimento pré-hospitalar de suporte avançado à vida [tese]. São Paulo: Escola de Enfermagem, Universidade de São Paulo; 2005. 